\title{
44504 - PROPOFOL REDUCES HYDROGEN PEROXIDE INDUCED CARDIAC AND ENDOTHELIAL CELL APOPTOSIS: ROLE OF AKT SURVIVAL SIGNALING PATHWAY
}

\author{
David Ansley, University of British Columbia Dept of Anesthesia, Vancouver, BC, Canada; \\ Baohua Wang, UBC Dept of Anesthesiology, Pharmacology and Therapeutics; \\ Tao Luo, UBC Dept of Anesthesiology, Pharmacology and Therapeutics;
}

INTRODUCTION: Endothelial nitric oxide (NO) produced by eNOS (endothelial nitric oxide synthase) is capable of inhibiting apoptosis and is regarded as a cell survival factor 1 . The phosphatidyl inositol 3-kinase (PI3K)/Akt cell survival pathway is able to regulate its expression and activation 2. We tested the hypothesis that propofol modulates Akt activated endothelial nitric oxide synthase expression to confer protection in endothelial cells and cardiomyocytes exposed to oxidative stress.

METHODS: Cultured human umbilical vein endothelial cells (ATCC, CRL1730) and Rat H9c2 cardiac myoblasts (ATCC, CRL1446) were used in an experimental model where hydrogen peroxide (H2O2) was used as the stimulus of oxidative stress. Study groups included 1) control, 2) cultured cells incubated with $\mathrm{H} 2 \mathrm{O} 2$ (100 \&\#956; M for endothelial cells and 400 \&\#956; $\mathrm{M}$ for cardiac myoblasts) alone, 3) cells incubated with propofol (50 \&\#956;M) alone, or 4) cells pretreated with propofol 50 \&\#956; M for thirty minutes then co-incubated with $\mathrm{H} 2 \mathrm{O} 2$. Cell viability was assessed using 3-(4,5-dimethylthiazol-2-yl)-2,5-diphenyltetrazolium bromide (MTT) assay. Cell apoptosis was evaluated by Hoechst 33258 staining. Activity of caspase-3 was determined by the colorimetric CaspACE ${ }^{\mathrm{TM}}$ Assay System (Promega, USA). Expressions of total Akt, phospho-Akt and endothelial nitric oxide synthase (eNOS) were detected by Western blotting.

RESULTS: $\mathrm{H} 2 \mathrm{O} 2$ decreased cell viability, induced cell apoptosis and increased caspase-3 activity in both endothelial cells and cardiomyocytes. $\mathrm{H} 2 \mathrm{O} 2$ did not cause significant change in eNOS expression. Propofol significantly protected cells from $\mathrm{H} 2 \mathrm{O} 2$-induced cell damage and apoptosis. Propofol significantly decreased caspase-3 activity, increased eNOS expression, but did not change phospho-Akt expression in endothelial cells. Propofol significantly decreased caspase- 3 activity and enhanced phospho-Akt expression in cardiomyocytes compared to $\mathrm{H} 2 \mathrm{O} 2$ stimulated cells.

CONCLUSIONS: The phenolic antioxidative and caspase-3 related antiapoptotic effects of propofol indicate its pleiotropic nature, acting differently on endothelial cells and cardiomyocytes via Akt-independent vs Akt-dependent pathways, respectively. We provide possible mechanistic support for the use of propofol as a preemptive cardioprotectant for cardiac surgery, where elevated oxidant stress may preexist (eg. diabetes).

Reference

1. Dimmeler, S. and Zeiher, A.M. Nitric oxide-an endothelial cell survival factor. Cell Death Differ 1999 6:964-8. 
2. Abdallah, Y. and Schafer, C. Insulin: an overall cardiovascular protector? Cardiovasc Res 2006 69:4-6. 